\title{
Effects of adipose stem cell sheets on colon anastomotic leakage in an experimental model: Proof of principle
}

Panithi Sukho, DVM, ${ }^{\mathrm{a}, \mathrm{b}, \mathrm{c}}$ Geesien S.A. Boersema, MD, ${ }^{\mathrm{d} *}$ Abigael Cohen, $M D,{ }^{e}$ Nicole Kops, BS, ${ }^{e}$ Johan F Lange, $M D$, PhD, ${ }^{d}$ Jolle Kirpensteijn, DVM, PhD, , f, Jan Willem Hesselink, DVM, PhD, ${ }^{a}$ Yvonne M. BastiaansenJenniskens, PhD, ${ }^{\mathrm{e}}$ Femke Verseijden, DVM, PhD, ${ }^{\mathrm{a}, \mathrm{e} * *}$

a Department of Clinical Sciences of Companion Animals, Faculty of Veterinary Medicine, Utrecht University, Utrecht, The Netherlands

b Department of Otorhinolaryngology, Erasmus MC University Medical Center, Rotterdam, The Netherlands

c Department of Clinical Sciences and Public Health, Faculty of Veterinary Science, Mahidol University, Nakhon Pathom, Thailand

d Department of Surgery, Erasmus MC University Medical Center, Rotterdam, The Netherlands

e Department of Orthopaedics, Erasmus MC University Medical Center, Rotterdam, The Netherlands

f Hills Pet Nutrition Inc, Topeka, Kansas, USA

* shared 1st author

${ }^{* *}$ Corresponding author: f.verseijden@uu.nl 


\section{ABSTRACT}

The most dreaded complication of colorectal surgery is anastomotic leakage. Adipose tissue-derived stem cell sheets (ASC sheets) prepared from temperatureresponsive culture surfaces can be easily transplanted onto tissues. These sheets are proposed to improve cell transplant efficiency and enhance wound healing. The aim of this study was to investigate whether application of ASC sheets could prevent leakage of sutured colorectal anastomoses. Insufficient suturing of colorectal anastomoses was performed in Wistar rats to create a colorectal anastomotic leakage model. Rats were randomized to ASC sheet application or control group. Leakage, abscess formation, adhesion formation, anastomotic bursting pressure (ABP), and histology were evaluated on postoperative day 3 or 7. ASC sheet application significantly reduced anastomotic leakage compared to controls, without increased adhesion formation. ASC sheet transplantation resulted in more CD3+ T-cells and CD163+ anti-inflammatory macrophages at the anastomotic site than the control group. ABP, vessel density and collagen deposition were not different between groups. Using cell sheet technology, we generated ASC sheets that prevented disruption of sutured colorectal anastomoses as shown by reduced leakage. Increased numbers of anti-inflammatory macrophages and T-cells might have contributed to this positive effect.

Keywords: Adipose tissue-derived stem cell, cell sheet, anastomotic leakage, healing 


\section{INTRODUCTION}

Gastrointestinal (GI) disease is the third most common cause of death, the leading cause of cancer death, and the most common cause of hospital admission (Source: British Society of Gastroenterology, clinical services 2007). In 2009, the US National Institutes of Health reported 20 million ambulatory surgical GI procedures, to resect tumors, to relieve obstruction, after trauma, or as part of bariatric surgery [1]. An important and life-threatening complication of GI surgery is anastomotic leakage. Despite years of research, the incidence of impaired wound healing and consequent leakage remains high, ranging from $3 \%$ in small bowel surgery to $25-27 \%$ in colorectal surgery with mortality rates of up to $22 \%[2,3]$. Anastomotic leakage has a multifactorial etiology. Besides technical failures, restricted blood supply and uncontrolled inflammation are important reasons that contribute to impaired wound healing $[4,5]$.

Studies using human adipose tissue-derived mesenchymal stem cells (ASCs) have been successful in regenerating various tissues and promoting wound healing [6]. More importantly, several reports indicate that ASCs help to ameliorate tissue inflammation and can accelerate new blood vessel formation. These capacities seem among others to be attributable to the ability of ASCs to secrete a myriad of growth factors and cytokines that can promote repair of injured tissue or improve the quality of tissues that are regenerated [7, 8]. The beneficial characteristics of ASCs could be exploited to promote the healing process in the intestinal wall after surgery, thus helping to prevent postoperative complications. Recent studies using intraperitoneal ASC injections or ASC-coated bio-sutures showed no prevention from anastomotic leakage [9, 10]. Injection of ASCs as suspension or combined with biomaterials such as fibrin, collagen or gelatin is either associated with cell washout, insufficient cell retention or an inadequate distribution of transplanted cells.

Currently, cell sheet technology is a promising technique for cell transplantation improving cell retention. Cell sheets are typically prepared on special culture dishes that are coated with a temperature-responsive polymer. This polymer changes from being hydrophobic to hydrophilic when the temperature is lowered. In this way, intact cell sheets can be removed as one piece from the culture dishes without enzymatic treatment, preventing destruction of cellular and cell-extracellular matrix interactions within the sheet $[11,12]$.

Despite the application of various tissue sealants and other biomaterials, no significant reduction in the incidence of anastomotic leakage has been accomplished over the last 30 years [13]. Clearly, there is a need for new strategies therefore we propose a novel approach in which ASCs are applied to the surgical wound area in cell sheets that are permeable to growth factors secreted by the ASCs and can be handled with ease by the surgeon. Therapeutic potential of ASC sheets was shown

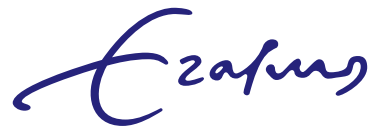


to improve cardiac tissue regeneration in the treatment of myocardial infarction [14], dilated cardiomyopathy [15], healing of hind limb ischemia [16] and chronic non-healing skin wounds [17]. Since cell sheet technology has been successfully used in improving healing of other soft tissues (i.e., heart, skin) as we mentioned above, we explored whether ASC sheets could serve as an ideal approach for the local delivery of cells to enhance healing and prevent leakage after intestinal anastomosis. To our knowledge this is the first attempt of ASC sheet application on intestinal surgical wounds.

The aim of this proof of principle study is to validate the efficacy of ASC sheets to prevent anastomotic disruption and leakage 3 and 7 days postoperatively in a colorectal leakage model in rats.

\section{MATERIALS AND METHODS}

\section{ASC sheet preparation}

ASCs were isolated from subcutaneous abdominal adipose tissue (all women, mean age $40 \pm 9$ years old). ASCs were seeded at 400,000 cells $/ \mathrm{cm}^{2}$ on $100 \% \mathrm{FBS}$ pre-coated temperature responsive dishes (3.5 cm diameter, CellSeed, Tokyo, Japan). Every single sheet was made from one ASCs donor. ASCs were cultured in Dulbecco's Modified Eagle Medium 1 g/l glucose (LG-DMEM, Gibco, Life technologies, Paisley, UK) supplement with 10\% fetal bovine serum (FBS, Lonza, Verviers, Belgium) with $50 \mu \mathrm{g} / \mathrm{ml}$ gentamicin (Gibco) and $1.5 \mu \mathrm{g} / \mathrm{ml}$ fungizone ${ }^{\circledR}$ (Gibco). After 48 hours, ASCs formed a coherent cell sheet. Thirty minutes before in vivo application, culture dishes were placed at room temperature to enable spontaneous detachment of the ASC sheets then culture medium was removed and refreshed with serum-free medium.

\section{ASC sheet viability and histology in vitro}

ASC sheet viability was evaluated by fluorescence microscopy on 0, 3, and 6 hours after detachment using a live/dead staining (Invitrogen, Life Technology, foster City, CA, USA). Briefly, ASC sheets were washed with Phosphate Buffered Saline (PBS, Gibco) after detachment and incubated for 30 minutes in calcein AM dye 1 $\mu \mathrm{l} / \mathrm{ml}$ and ethidium bromide dye $1.5 \mu \mathrm{l} / \mathrm{ml}$ at $37^{\circ} \mathrm{C}$ in a humid atmosphere with $5 \% \mathrm{CO}_{2}$. The sheets were analyzed with fluorescence microscopy using Olympus IX71 inverted microscope, and images were captured with Cell F Imaging Software (Olympus: Hamburg, Germany, 2008). ASC sheets were fixed overnight in $4 \%$ buffered formaldehyde (Sigma, St Louis, Missouri and Merck, Billerica, Massachusetts), set in in $2 \%$ agarose (Eurogentec, Liege, Belgium) and embedded in paraffin. Cross sections were stained with hematoxylin and eosin (H\&E, Sigma).

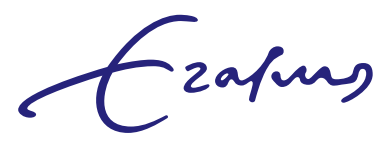




\section{SPIO-labeling of ASC sheet for pilot experiment}

In a pilot study, ASCs were labeled with the so-called superparamagnetic iron oxide particles (SPIO) using ferumoxides (Endorem, Guerbet S.A., Paris, France) complexed to protamine sulphate (LEO Pharma N.V., Wilrijk, Belgium) as previously described [18]. For removal of extracellular iron, cells were washed with PBS containing heparin $10 \mathrm{U} / \mathrm{ml}$ (LEO Pharma B.V., Breda, the Netherlands). SPIO labeling mix was made at a constant concentration of $100 \mu \mathrm{g} / \mathrm{ml}$ ferumoxides with $5 \mu \mathrm{g} / \mathrm{ml}$ protamine to ensure identical particle formation. SPIO-labeled ASC were combined with unlabeled ASCs in a 30:70 ratio to form ASC sheets. Labeled ASC sheets were transplanted to 6 pilot rats after colorectal anastomosis to examine ASC sheet survival at POD3 $(n=4)$ and $7(n=2)$. Only for this pilot study, ASCs were labeled. In the following experiments, unlabeled ASCs were used to avoid any unwanted effects of labeling on ASC sheet function.

\section{Animals}

Male Wistar rats (weight 250-350g) were purchased from a licensed breeder (Harlan Laboratories, Boxmeer, The Netherlands). All rats were bred under specific pathogen-free conditions and randomly kept under standard laboratory conditions. Standard rat chow and water were supplied ad libitum. The research protocol was approved by the Ethical Committee of Animal Experimentation, Erasmus University Rotterdam (133-14-01). Sixty rats were randomly allocated to 4 experimental groups. The ASC sheet groups received local application of an ASC sheet around the colorectal anastomosis, anastomotic healing being evaluated on postoperative day $3(n=15)$ or $7(n=15)$. Rats in the control groups received no ASC sheet and anastomotic healing was evaluated at postoperative day $3(n=15)$ or $7(n=15)$.

\section{Surgical technique and application of ASC sheets}

To evaluate the ability of ASC sheet to prevent leakage the colorectal anastomotic leakage rat model previously described by Wu et al. was used [19]. Briefly, the rats were anesthetized with isoflurane/oxygen inhalation, and $0.05 \mathrm{mg} / \mathrm{kg}$ buprenorphine intramuscularly. After aseptic preparation, a midline abdominal incision was made and the right, middle, and left colic arteries were ligated (Silkam 4/0; B Braun, Melsungen, Germany). The colonic segment was resected $1 \mathrm{~cm}$ aborally to the cecum and $0.5 \mathrm{~cm}$ above the caudal mesenteric artery. An insufficiently sutured end-to-end anastomosis was created by one-layer inverting suturing with 5 interrupted sutures (Dafilon 8/0, B Braun) (Fig. 1A). Rats were randomly divided into control or ASC sheet groups. To make sure the same side of the sheet was always applied and to avoid variation because of different orientation of the sheets, the dish-side of cell sheet was always applied to the serosal surface of the colon in every rat. In the ASC sheet group, the dish-side of an ASC sheet was wrapped

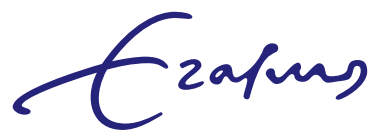


around the anastomosis line (Fig. 1B). The ASC sheet adhered spontaneously to the serosal surface of the colon and required no further suturing or glue. The control group received no other intervention except for the insufficiently sutured end-toend colorectal anastomosis. Immediately after ASC sheet application, the abdomen was closed in two layers of running sutures (Safil 5/O, B. Braun). Immediately postoperatively, rats were rehydrated and warmed. The rats returned to a normal diet after recovery from surgery.

A
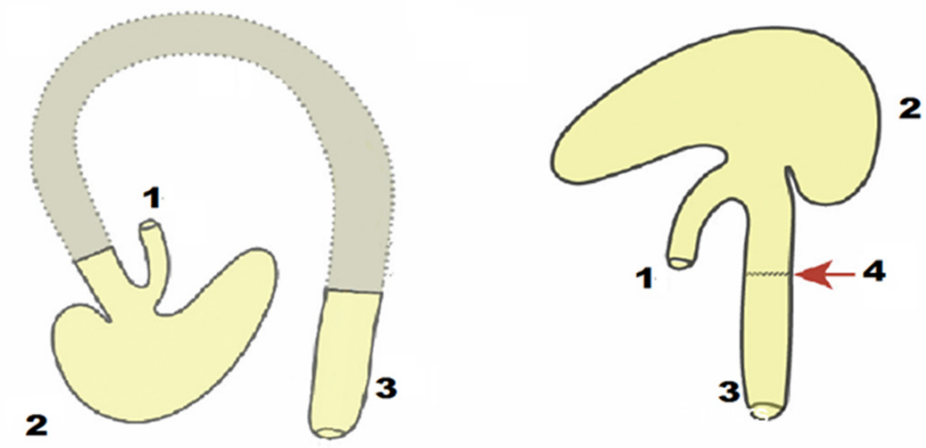

B
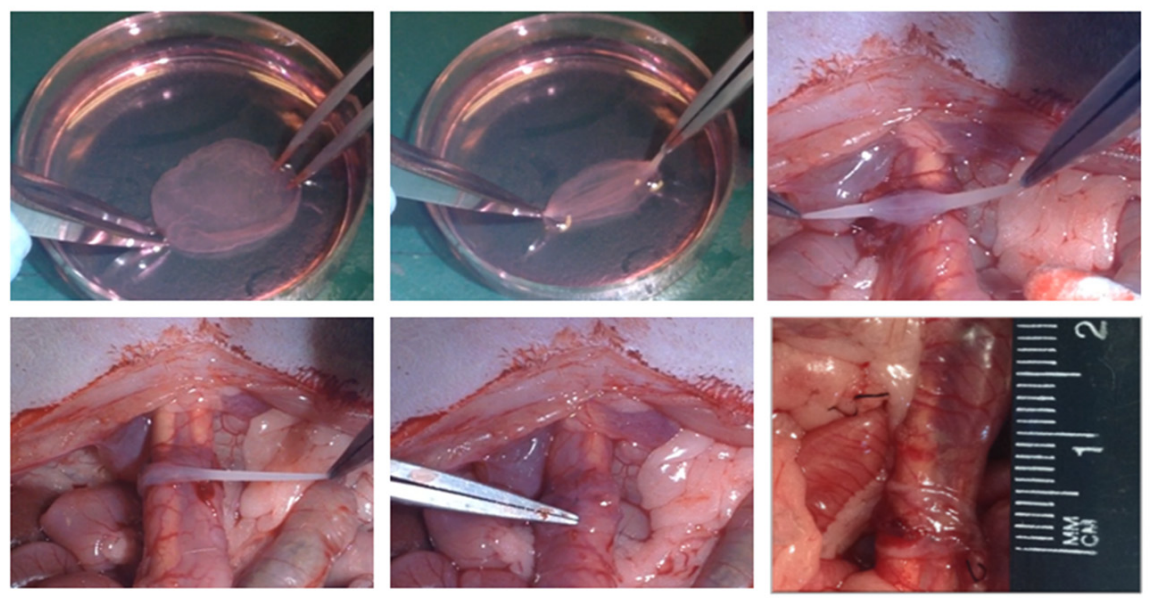

Figure 1. Rat partial colectomy and ASC sheet application.

(A) Schematic overview of the rat colon before and after partial colectomy*. Anatomy: (1) terminal ileum, (2) cecum, (3) rectum and anus, (4) anastomosis. (B) ASC sheet transplantation (from upper left to lower right); ASC sheets were grasped with forceps at two rims and applied on top of the colorectal anastomosis. Following application, the sheet was unfolded and wrapped around the anastmosis thereby ensuring to cover it completely. ${ }^{*}$ The schematic overview was adapted with permission from Wu Z, et al. Reducing colorectal anastomotic leakage with tissue adhesive in experimental inflammatory bowel disease. Inflamm Bowel Dis. 2015; 21(5):1038-46. Copyright@ 2016 copyright Clearance center, Inc. 


\section{Follow up}

Since both undifferentiated and differentiated ASCs have been used successfully for xenotransplantation in different other applications [20], no immunosuppressive drugs were used in this experiment. Wellness and weight of all rats were evaluated daily. On postoperative day (POD)3 or 7, rats were re-anesthetized and a re-laparotomy was performed. The abdomen was checked for signs of peritonitis, adhesions and abscesses (defined as "elsewhere"). At the anastomotic area, signs of stricture, disruption, adhesion, and abscesses were checked. Two observers (PS and GB) performed all macroscopic observations and evaluations for each rat.

\section{Macroscopic observation}

Abscess severity was scored according to the previously described abscess score, in short: $0=$ no abscess, $0.5=$ one small abscess $(<1 \mathrm{~mm}), 1=$ several small abscesses, $2=$ medium abscess $(1-3 \mathrm{~mm}), 3=$ one large $(3-5 \mathrm{~mm})$ or several medium abscesses, 4 = one very large $(>5 \mathrm{~mm}$ ) or several large abscesses [21]. Adhesions were recorded using the Zühlke score. In short: 0 = no adhesions, 1 = firm adhesions which can be separated with blunt dissection, 2 = strong adhesions which can only be separated with sharp dissection, 3 = very strong vascularized adhesions which can only be separated with sharp dissection and damage to surrounding tissue is hardly preventable. Anastomotic bursting pressure $(\mathrm{ABP})$ was determined after macroscopic observation by a third blinded researcher (AC). In short, $A B P$ was determined by insufflation of air in a closed segment of the colon and pressure at time of the first air leak was noted as bursting pressure. The location of the burst was also noted. After the ABP measurement, the colorectal anastomosis was resected and prepared for histological examination.

\section{Histological evaluation after transplantation}

Histological and immunohistochemically assessment were conducted by two independent blinded observers (PS and AC) in a fully randomized order to evaluate healing of the anastomosis. A segment of colon containing the colorectal anastomosis was harvested, washed with phosphate-buffered saline and opened longitudinally. After overnight fixation in $4 \%$ buffered formaldehyde, segments were embedded in paraffin. Paraffin-embedded sections $(6 \mu \mathrm{m})$ were deparaffinized and rehydrated. For morphological evaluation, sections were stained with H\&E. Perl's iron stain (Klinipath BVBA, Duiven, the Netherlands) was used for staining SPIO-labeled ASC sheets at POD3 and POD7 after transplantation in the pilot study. Picro Sirius red staining (Sigma, St Louis, Missouri) was used for collagen deposition. Immunohistochemistry was performed to assess vascular density (rat, CD34+), presence of T-cells (CD3+) and anti-inflammatory M2 macrophages (CD163+), ASC sheet survival (human mitochondria) and endothelial differentiation (human CD31+) of

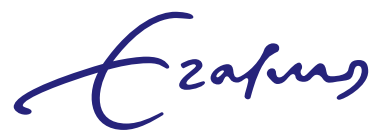


ASCs at the colorectal anastomosis. Details of the methods used for staining are described in supporting information.

\section{Statistical analysis}

Data are presented as mean \pm standard deviation (SD) and analyzed with the Mann-Whitney U test and Kruskal-Wallis one-way analysis of variance. To compare the incidence of anastomosis disruption between groups at each time point the two-tailed Chi-squared test was used. All reported $P$ values are two-sided; a $P$ value of $<0.05$ indicated statistical significance. All statistical analyses were done using SPSS 21.0 (IBM Inc., Armonk, NY, USA).

\section{RESULTS}

\section{ASC sheet characteristics in vitro}

ASCs cultured at 400,000 cells $/ \mathrm{cm}^{2}$ in a temperature responsive culture dish for 48 hours formed cell sheets that could be spontaneously detached at room temperature (Fig. 2A). ASC sheets consisted of several cell layers (Fig. 2B). Viability of ASC
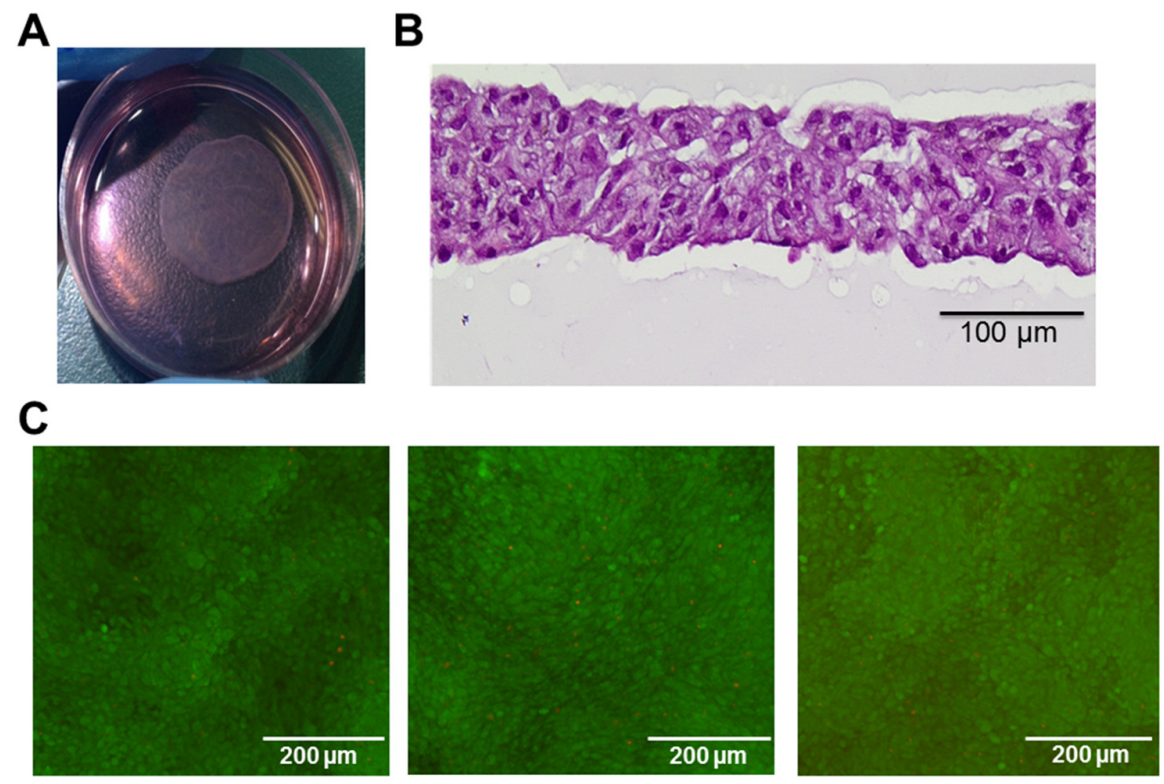

0 hour

3 hours

6 hours

Figure 2. ASC sheet viability and morphology prior to implantation.

(A) ASC sheet after detachment from the temperature responsive dish. (B) Cross section of ASC sheet stained with hematoxylin \& eosin. (C) Live/dead staining of ASC sheets at 0, 3 and 6 hours after detachment. Viable cells stained with calcein (green), non-viable cells stained with ethidium bromide (red).

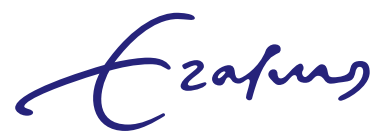


sheets in medium at room temperature remained high until 6 hours after detachment (Fig. 2C).

\section{In vivo overall observations}

During the first postoperative days, all rats lost weight and had a lower wellness score than before surgery. From POD4 onwards, all animals started to gain weight. The weight loss and wellness scores in the control and ASC sheet groups were not significantly different (supplementary data; Fig. S1). Three out of 60 rats (5\%) died within 24 hours after surgery in both control (POD3) and ASC sheet groups (POD3 and POD7). Autopsy demonstrated that these deaths were caused by acute (e.g., bleeding and anesthesia-related) complications; therefore, these animals were excluded from further analysis.

\section{Postoperative intra-abdominal evaluation}

At re-laparotomy, the ASC sheet structure was visible around the anastomosis in all rats of the ASC sheet group at POD3 and POD7 (Fig 3A-B). Ten out of 14 rats in the control group had anastomotic disruption on POD3 (71\%) versus 2 out of 14 (14\%) in the ASC sheet group $(P=0.002)$. No significant difference in the anastomotic disruption rate was observed at POD7 between the two groups. Significantly more rats in the control group had abscesses at POD7 around the anastomosis (10/15: $67 \%)$ compared to the group receiving an ASC sheet (4/14: $28 \%, P=0.04)$. There were no differences in the occurrence of peritonitis, stricture formation, adhesions, and abscess formation elsewhere (Table 1). The average abscess number around the anastomosis was significantly higher in the control group than in the ASC sheet group on both POD3 $(1.6 \pm 0.5$ versus $1.0 \pm 0.7, P=0.004)$ and POD7 $(1.2 \pm 1.2$ versus $0.4 \pm 0.6, P=0.028)$, (Fig. 4A). The abscess score between the ASC sheet and control groups did not differ on POD3 but the abscess score of the ASC sheet group was
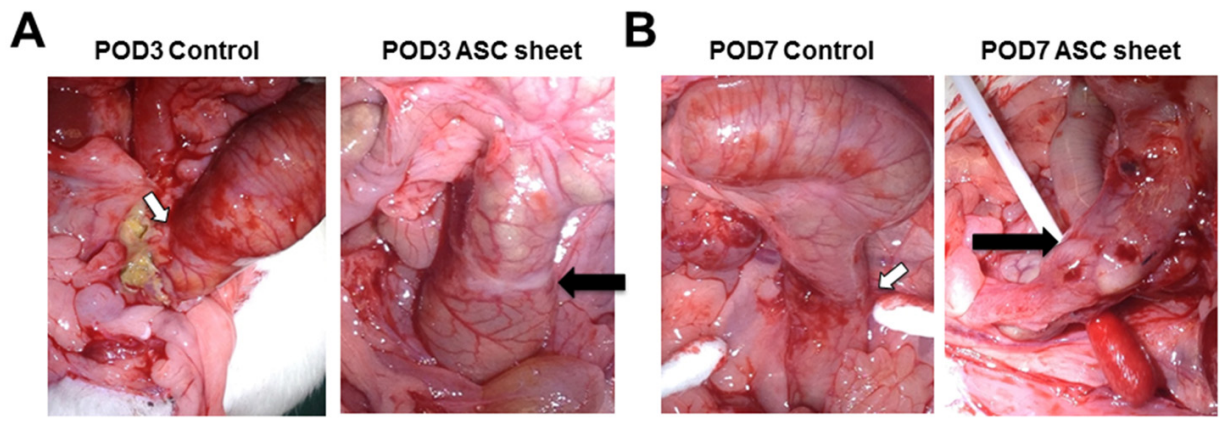

Figure 3. ASC sheet is visible around the anastomosis.

(A) POD3 and 7 (B), white arrows point at anastomotic area and black arrows point at transplanted ASC sheets.

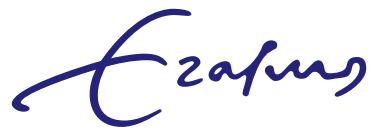


Table 1. Post-operative macroscopic findings

\begin{tabular}{lllllll}
\hline & \multicolumn{7}{c}{ POD3 } & & \multicolumn{2}{c}{ POD7 } \\
\hline & Control (\%) & ASC sheet (\%) & $\boldsymbol{p}$-value & Control (\%) & ASC sheet (\%) & $p$-value \\
Peritonitis & $1 / 14(7.1)$ & $0 / 14(0)$ & NS & $0 / 15(0)$ & $0 / 14(0)$ & NS \\
Anastomotic disruption & $10 / 14(71.4)$ & $2 / 14(14.3)$ & 0.002 & $3 / 15(20)$ & $2 / 14(14.3)$ & NS \\
Stricture & $2 / 14(14.3)$ & $2 / 14(14.3)$ & NS & $2 / 15(13)$ & $2 / 14(14.3)$ & NS \\
Abscess at anastomosis & $14 / 14(100)$ & $12 / 14(85.7)$ & NS & $10 / 15(66.7)$ & $4 / 14(28.6)$ & 0.04 \\
Adhesion at anastomosis & $14 / 14(100)$ & $14 / 14(100)$ & NS & $15 / 15(100)$ & $14 / 14(100)$ & NS \\
Abscess elsewhere & $11 / 14(78.5)$ & $8 / 14(57.1)$ & NS & $6 / 15(40)$ & $4 / 14(28.6)$ & NS \\
Adhesion elsewhere & $8 / 14(57.1)$ & $3 / 14(21.42)$ & NS & $9 / 15(60)$ & $7 / 14(50)$ & NS \\
\hline
\end{tabular}

Values are presented as the number of rats and relative percentage. POD3= postoperative day $3, \mathrm{POD} 7=$ postoperative day 7. ASC = adipose tissue derived stem cell. NS; not significant by Chi-square test.

A

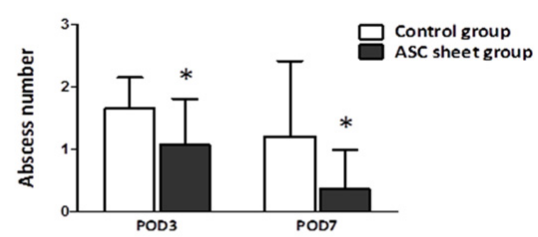

Adhesion number

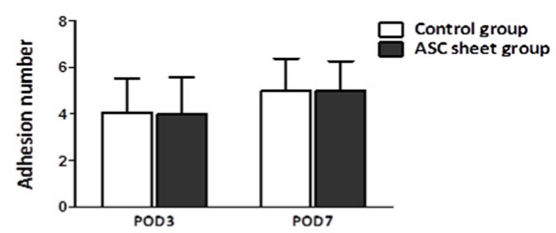

E Anastomotic bursting pressure

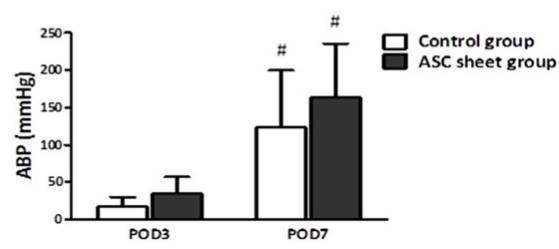

B Abscess score

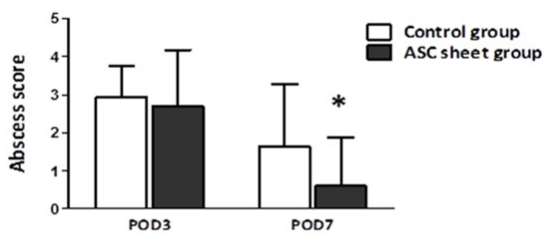

D Adhesion score

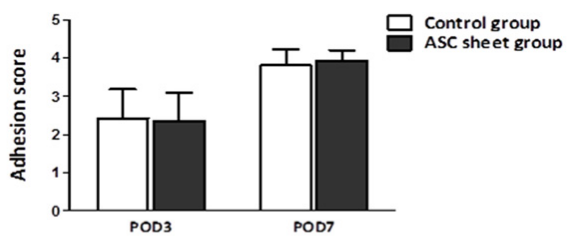

Figure 4. Intra-abdominal macroscopic findings at colorectal anastomosis.

(A) abscess number, (B) abscess score (O-4), (C) adhesion number, (D) adhesion score (O-4) and (E) anastomotic bursting pressure (ABP). ASC = adipose tissue-derived stem cells. $\mathrm{POD} 3=$ postoperative day 3 . POD7 = postoperative day 7. Each bar represents average number $+/$ - standard deviation from control ( $n=14$ in the control and ASC sheet group at POD3; $n=15$ in the control group and $n=14$ in the ASC sheet group at POD7). ${ }^{*} P<0.05$ between groups. $\# P<0.05$ between different time points within the same group. 
significantly lower than the control group on POD7 ( $P=0.048$, Fig. 4B). The number of intra-abdominal adhesions was significantly lower in the ASC sheet group $(P=$ 0.043 ) on POD3 (supplementary data, Fig. S2), but no significant differences in the number of adhesions (Fig. 4C) and adhesion scores (Fig. 4D) around the anastomosis were seen between the two groups on POD3 and POD7.

On POD7 the average bursting pressure was higher compared to POD3 in both groups but was not significantly different between groups (Fig. 4E). On POD3 bursting of all anastomoses in both the control and ASC sheet groups occurred at the anastomosis line. On POD7 bursting occurred predominantly at the anastomosis line in the control group (10 rats $(66 \%)$, whereas in the ASC sheet group bursting mostly (8 rats: $57 \%$ ) occurred proximally or distally to the anastomosis line.

\section{Histological evaluation}

To evaluate whether ASC sheets would still be present 3 and 7 days after transplantation, Perl's iron stain was used for detection of SPIO-labeled ASC sheets. At both POD3 and POD7, SPIO-labeled ASCs were present at the colorectal anastomotic serosal side. (Fig. 5A, B). Further evaluation of ASC sheet state was performed by using a human mitochondrial staining. At POD3 ASC sheets showed abundant positive staining for human mitochondria, while at POD7 positive cells were severely diminished (Fig. 6A,B).

To evaluate the effect of the ASC sheets on colorectal anastomosis vascularization, collagen deposition and infiltration of inflammatory cells, several specific immunohistochemically stainings were performed. Local application of ASC sheets did not affect $\mathrm{CD} 34$ positive capillaries or collagen deposition at the colorectal anastomosis site. Although the capillary density increased from POD3 to POD7 in both the control and ASC sheet groups $(P<0.01)$, there was no difference in the capillary density between groups at either time points (POD3, ASC sheet group 172.4 \pm 44 vessels $/ \mathrm{cm}^{2}$ versus control group $150.5 \pm 42$ vessels $/ \mathrm{cm}^{2}$ and POD7, ASC sheet group $277.2 \pm 95$ vessels $/ \mathrm{cm}^{2}$ versus control group $276.5 \pm 83$ vessels $/ \mathrm{cm}^{2}$, Fig. 7A,E). No human ASC-derived CD31 positive endothelial cells were detectable within the ASC sheets before or after transplantation (data not shown).

Similar findings regarding collagen deposition at the anastomotic site were found. Despite an increase of collagen deposition from POD3 to POD7 in both groups, groups were not significantly different from each other (POD3, ASC sheet group $2.2 \pm 2.4 \%$ versus control group $0.7 \pm 0.6 \%$, and POD7, ASC sheet group $19.5 \pm$ $0.6 \%$ versus control group $22.7 \pm 10.5 \%$, Fig. $7 \mathrm{~B}, \mathrm{~F})$.

The number of T-cells and M2 macrophages was used to evaluate the inflammatory response at the anastomosis site. At POD3 a nonsignificant different number of CD3 positive T-cells were found in the ASC sheet group ( $81.8 \pm 36$ cells/ $\left.\mathrm{cm}^{2}\right)$ as compared to the control group $\left(103.6 \pm 45 \mathrm{cells} / \mathrm{cm}^{2}\right)$. The number of CD3

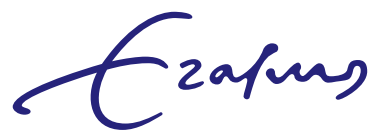


A
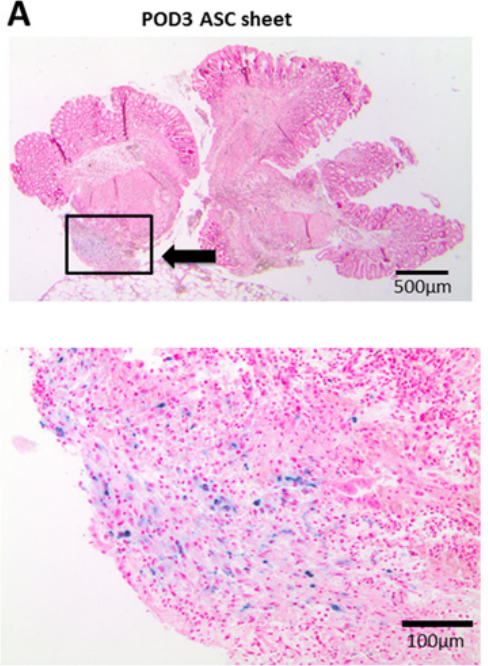

B
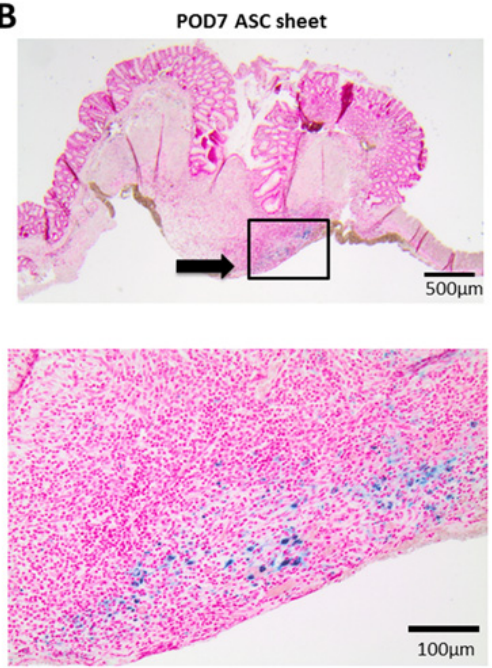

Figure 5. Tracking SPIO-labeled ASC sheet in a pilot experiment.

SPIO-labeled ASC sheet stained with Perl's iron stain (blue). The selected area with solid line (see also black arrows) indicates the anastomotic area, which is enlarged and illustrated below for representation of positively stained cells A-B. Blue iron stained SPIO-labeled ASCs present in ASC sheet on serosal side of the colorectal anastomosis at (A) POD3 and (B) POD7.

A
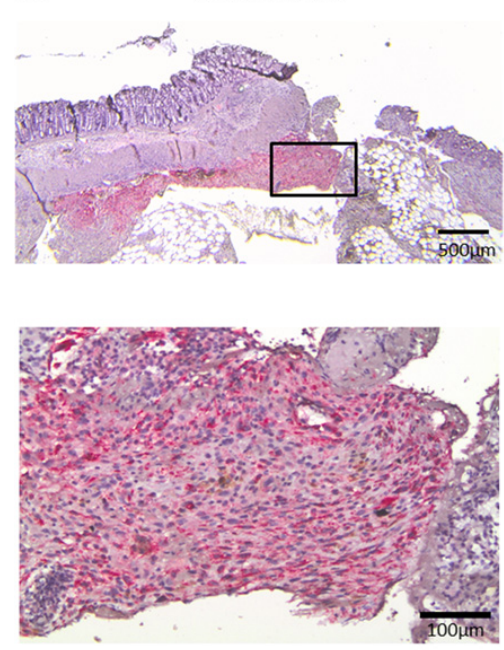

B POD7 ASC sheet
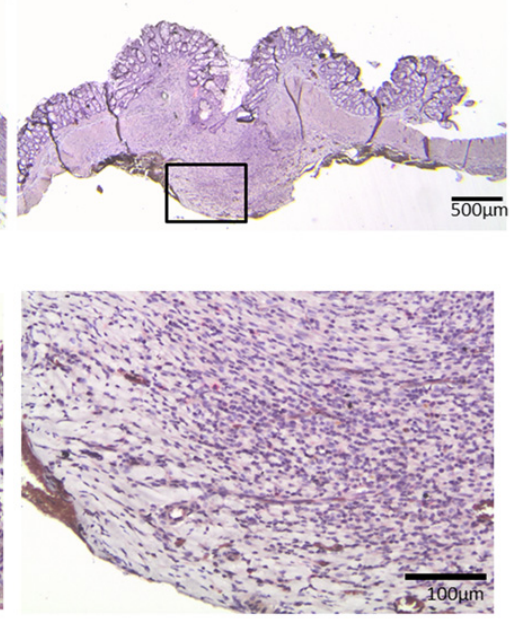

Figure 6. ASC sheet survival at anastomotic site.

The selected area with solid line indicates the anastomotic area with ASC sheet, which is enlarged and illustrated below for representation of positive staining for human mitochondria A-B. (A) ASC sheet showed positive red staining of human mitochondria at POD3 and (B) severely diminished positive staining at POD7 

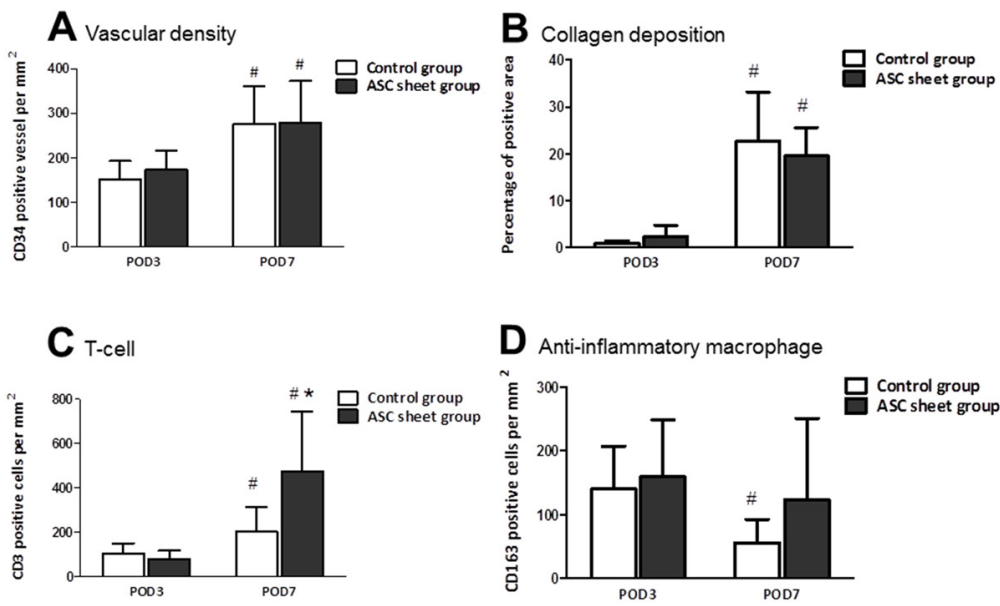

D Anti-inflammatory macrophage
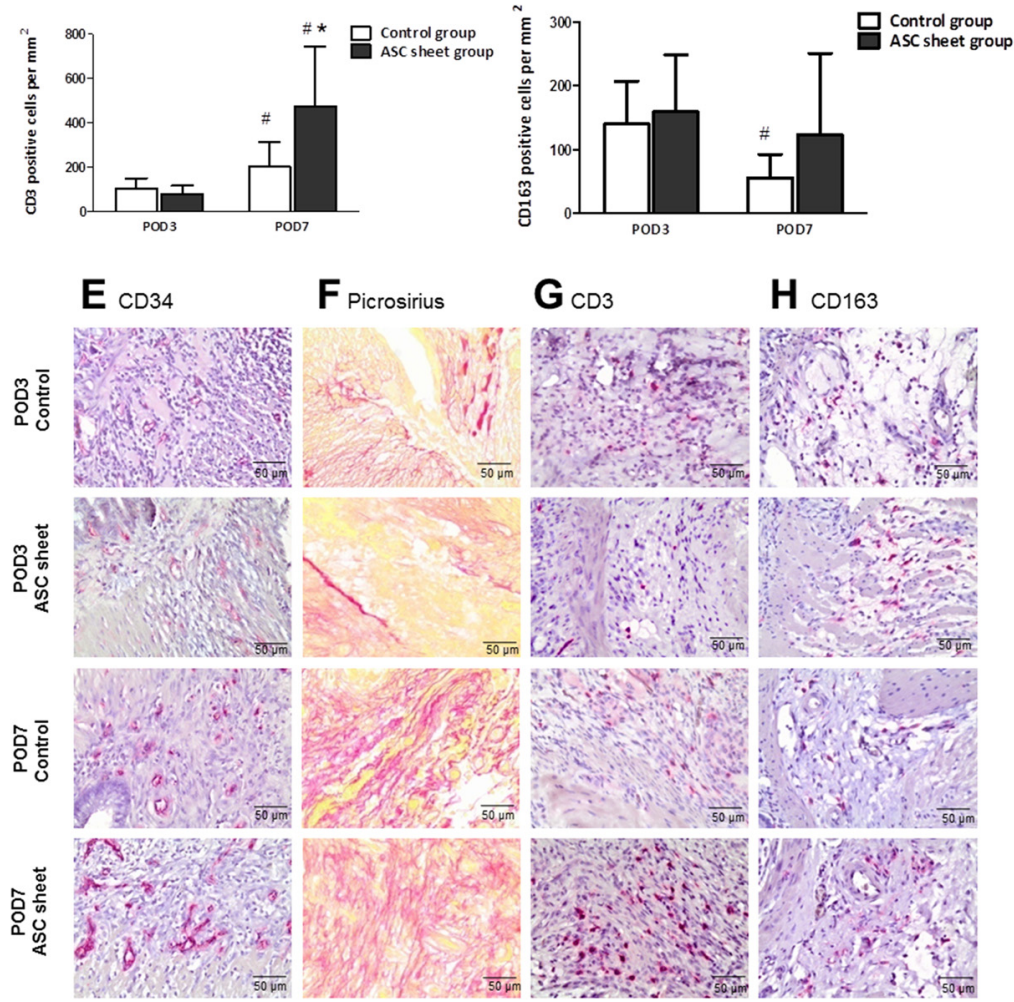

Figure 7. Comparison of vascular density, collagen deposition, T-cell and anti-inflammatory macrophage amount at the anastomotic site on POD3 and POD7 between ASC sheet and control group. The comparisons were listed in A-D and illustrated in $\mathrm{E}-\mathrm{H}$.

(A) Vascular density as assessed by average number of CD34+ vessels $/ \mathrm{mm}^{2}$. (B) Collagen deposition determined by area percentage of Picrosirius red staining. (C) T-cell response determined by number of $\mathrm{CD} 3+$ cells $/ \mathrm{mm}^{2}$. (D) Anti-inflammatory macrophage response assessed by average CD163+ cells $/ \mathrm{mm}^{2}$. POD3 = postoperative day 3. POD7 = postoperative day $7 . A S C=$ adipose tissue-derived stem cells. Each bar represents average value +/- standard deviation from control $(n=14$ in the control and ASC sheet group at POD3; $n=15$ in the control group and $n=14$ in the ASC sheet group at POD7. ${ }^{*} P<0.05$ between groups, \# $P<0.05$ between different time points within the same group. Representative examples of immunohistochemically staining of colorectal anastomosis for (E) CD34, (F) Picrosirius red, (G) CD3, and $(H)$ CD163 of each group (200x). 
positive cells increased in both groups from POD3 to POD7. At POD7 a significantly higher number of CD3 positive T-cells was detected in the ASC sheet group (508.6 \pm 243 cells $\left./ \mathrm{cm}^{2}\right)$ than in the control group $\left(204.6 \pm 107 \mathrm{cells} / \mathrm{cm}^{2}, P=0.001\right.$, Fig. 7C, G).

In the control group, the number of $\mathrm{CD} 163$ positive cells indicating M2 macrophages decreased significantly between POD3 (140.3 \pm 67 cells $/ \mathrm{mm}^{2}$ ) and POD7 (55.9 \pm 37 cells $/ \mathrm{mm}^{2}, P<0.001$ ) whereas in the ASC sheet group, the number of CD163 positive cells did not differ between POD3 (159.6 \pm 89 cells $\left./ \mathrm{mm}^{2}\right)$ and POD7 (122.8 \pm 128 cells $/ \mathrm{mm}^{2}$, Fig. 7D, H).

\section{DISCUSSION}

Cell sheets are a recent development in regenerative medicine. They allow for the delivery of cultured cells and their deposited extracellular matrix and prevent cell loss associated with administering cells via injection or scaffold [22]. Previous studies demonstrated that ASC sheets show great promise in promoting the healing of several organ injuries. In this study, application of an ASC sheet to an insufficiently sutured rat colorectal anastomosis resulted in reduced anastomotic disruption and abscess formation. ASC sheets facilitated an increase in the number of antiinflammatory macrophages and T-cells at the anastomosis site, which might have contributed to this positive effect.

Application of ASC sheets to colorectal anastomosis is feasible since ASC sheets are flexible and spontaneously adhere to the serosal surface rapidly. This might be due to adhesive proteins present at the dish-side of ASC sheet that remain after harvesting from a temperature responsive dish [23]. ASC sheet survival after in vivo transplantation has been shown in other in vivo models and varied form 3 days [24] up to 8 weeks [25]. In our in vivo model ASCs were still present in the sheets at POD3 and 7 and mitochondrial staining was present until day 3. These findings indicate that ASC sheet were present and contained living cells for at least 3 days after transplantation allowing them to exert their potential paracrine effects for several days.

The recording of bursting pressure in colorectal anastomoses showed that on POD7, the average bursting pressure was not different between the groups. However, the bursting site in the ASC sheet group was mainly remote from the anastomotic line compared with the control group in which the burst occurred mainly at the anastomosis itself. These results suggest that when an intraluminal pressure is exerted to the colonic wall, anastomosis that is reinforced by ASC sheet is less susceptible to perforation. Alternative techniques that have been used previously in an attempt to prevent leakage of gastrointestinal anastomosis have been based on the sealing of the anastomosis with tissue adhesives or synthetic meshes $[26,27]$. Although these techniques for leakage prevention have various levels of

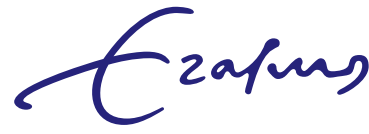


clinical efficacy, they are imperfect solutions that can give burdens for patients. The therapeutic potential of ASC sheets is generally thought to be attributable to ASCs paracrine ability, not the mechanical/physical strength of the ASC sheet to seal the wound [28-30]. Although ASC sheets are composed of only a thin layer of cells, it cannot be excluded that the reduction in dehiscence, and abscess formation in this study might be possible due to the mechanical ability of ASC sheets to seal the anastomosis and prevent leakage. However, merely external coating of colonic anastomoses has yet failed to show convincing results [31, 32]. Future experimental studies are warranted to determine and compare the mechanical role of external coating materials and ASC sheets for prevention of colon anastomotic leakage.

To investigate whether the ASC sheets contributed to the healing of the anastomosis, capillary density and collagen deposition were evaluated, both being of great importance for anastomotic healing. Although mesenchymal stem cells are believed to promote vascularization and stimulate collagen deposition, capillary density was not significantly different between the control and the ASC sheet groups in this model. Collagen deposition was also similar in the control and experimental groups on POD7. The lack of increased capillary density in the ASC sheet treated group conflicts with previously published data $[16,17]$ indicating that ASCs promoted angiogenesis in animal models characterized by low blood perfusion and ischemia such as hind limb ischemia [16], myocardial infarction, and skin wounds in diabetic rats [17]. However, no such effects were seen in a dilated cardiomyopathy model [15] or in gastric ulcer healing [33]. Possibly, the tissue to which the sheet was applied and whether or not this was ischemic might explain these differences. Transplanted human ASCs have been suggested to be able to differentiate into endothelial cells and thereby contributing to angiogenesis [34], however, this was not observed in the study presented here. The absence of increase in collagen deposition may indicate that although ASCs are able to produce collagen themselves [35] they do not promote collagen synthesis at the anastomosis site up to POD7. However, as suggested by Rabau et al [36] total collagen amount might be less important for anastomosis' tensile strength than structure and arrangement of collagen matrix.

Inflammatory cells play a key role in the healing of intestinal anastomoses [37]. Macrophages and activated lymphocytes are the main inflammatory cells in wound healing and in the development of fibrosis. Macrophages fulfil multiple roles in inflammation: besides phagocytosis and encouraging inflammation, macrophages also have important anti-inflammatory properties. Macrophages that stimulate inflammation are called M1 macrophages, whereas those that decrease inflammation and stimulate tissue repair are called M2 macrophages. Since the M2 subpopulation plays an important role in wound healing and ASCs have been shown to induce macrophage polarization towards the M2 subtype [38], we focused on the

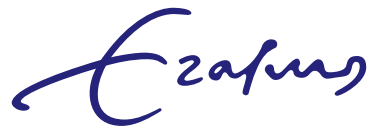


presence of M2 macrophages at the anastomosis area. As shown before in fetalmembrane mesenchymal stem cell sheets [39], the number of $M 2$ macrophages did not decline between POD3 and POD7 in the ASC sheet group when compared to the control group and might have stimulated the healing process at the anastomosis.

T-lymphocytes and more specific T-regulatory cells (T-regs), have the ability to release cytokines and growth factors that regulate other immune cells and positively affect wound healing [40]. On the other hand, in case of rejection of transplanted tissue, high numbers of cytotoxic T-cells are seen around the implanted tissue, together with abscesses and inflammation. In this study, the ASC sheet group had significantly more $\mathrm{CD} 3+\mathrm{T}$-cells within the anastomotic area than the control group at POD7, whereas the number of abscesses decreased instead of increased. Since the higher number of $\mathrm{CD} 3+\mathrm{T}$-cells at the anastomosis was accompanied with lower abscess number and anastomotic disruption, it is very unlikely that the increase of CD3+ T-cells is the results of rejection of the implanted ASC sheet. A more likely scenario is that the ASC sheets resulted in increased numbers of T-regs around the anastomosis thereby contributing to better healing of the wound. However, to our knowledge, a good staining for FoxP3, the classical marker for T-regs, is not available for rat at the moment.

Several studies suggest that human ASCs are immune-privileged and can survive in immunocompetent animals [41]. Both undifferentiated and differentiated ASCs have been used successfully for xenotransplantation [20]. Based on rat wellness score, and the positive effect of ASC sheets on colorectal anastomosis leakage we consider the presence of an (hyper)acute rejection in our study unlikely. However, future better understanding of the functionality of allo- or xenogeneicderived ASCs for therapy is warranted.

Unfortunately, it was not possible to fully blind the observers from the treatment group since the ASC sheet structure was obvious in macroscopic and microscopic evaluations. Moreover, we evaluated the effect of ASC sheets on colorectal anastomosis after POD3 and POD7 since dehiscence is most likely to occur in this critical phase of colonic anastomotic healing[13]. We are aware of the fact that at this time point the wound healing process is not complete yet and further maturation and collagen remodeling will occur at later stages of anastomotic healing [42].

The present study suggests that application of human ASC sheets to experimental colorectal anastomosis is a promising technique to prevent short-term post-operative complications such as anastomotic disruption and abscess formation without increasing adhesion formation. To translate the potential of ASC sheets for future clinical application, our results must be complemented with further short and long-term studies and defining the role of ASC sheets in the technique of colorectal anastomoses with regards to complications such as abdominal contamination and severe peritonitis.

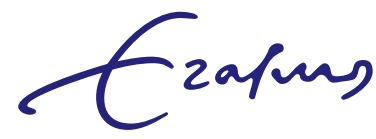




\section{CONCLUSIONS}

This study explored the proof of principle of a therapeutic potential of ASC sheet for preventing disruption of sutured colorectal anastomoses. ASC sheet application significantly enhanced healing of colorectal anastomoses with decreased incidence of disruption and abscess formation. The increased numbers of anti-inflammatory macrophages and T-cells might have contributed to promotion of the healing process. This preclinical study indicates that ASC sheet application may have a therapeutic role in promoting colorectal healing and prevention of anastomosisrelated complications.

\section{ACKNOWLEDGEMENTS}

The authors are grateful to Prof.Dr. S.E.R. Hovius, Dr.M.A.M. Mureau and all surgeons of the department of Plastic Surgery for the collection of subcutaneous adipose tissue. We are grateful to Maarten Leijs and Marianne Koolen for the kind supply of the SPIO-labeling and human mitochondria staining protocol, respectively.

\section{AUTHOR DISCLOSURE STATEMENT}

The authors declare that the research was conducted in the absence of any commercial or financial relationship that could be construed as a potential conflict of interest. P. Sukho is supported by a grant from The Netherlands Fellowship program (NFP-12/435), during the conduct of the study. Y.M. Bastiaansen-Jenniskens is supported by grants from Dutch Arthritis Foundation (LP11) and a Veni grant from NWO/STW. This study was performed within the Postgraduate School Molecular Medicine, Erasmus MC, University Medical Center, The Netherlands.

\section{REFERENCES}

[1] Kingham TP, Pachter HL. Colonic anastomotic leak: risk factors, diagnosis, and treatment. J Am Coll Surg. 2009;208:269-78.

[2] Snijders HS, Wouters MW, van Leersum NJ, Kolfschoten NE, Henneman D, de Vries AC, et al. Meta-analysis of the risk for anastomotic leakage, the postoperative mortality caused by leakage in relation to the overall postoperative mortality. European journal of surgical oncology : the journal of the European Society of Surgical Oncology and the British Association of Surgical Oncology. 2012;38:1013-9.

[3] Rullier E, Laurent C, Garrelon JL, Michel P, Saric J, Parneix M. Risk factors for anastomotic leakage after resection of rectal cancer. Br J Surg. 1998;85:355-8.

[4] Bruce J, Krukowski ZH, Al-Khairy G, Russell EM, Park KG. Systematic review of the definition and measurement of anastomotic leak after gastrointestinal surgery. Br J Surg. 2001;88:1157-68.

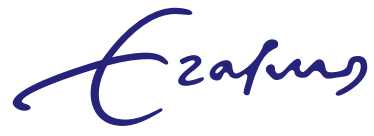


[5] Golub R, Golub RW, Cantu R, Jr., Stein HD. A multivariate analysis of factors contributing to leakage of intestinal anastomoses. J Am Coll Surg. 1997;184:364-72.

[6] Nakagami H, Morishita R, Maeda K, Kikuchi Y, Ogihara T, Kaneda Y. Adipose tissuederived stromal cells as a novel option for regenerative cell therapy. Journal of atherosclerosis and thrombosis. 2006;13:77-81.

[7] Hong SJ, Traktuev DO, March KL. Therapeutic potential of adipose-derived stem cells in vascular growth and tissue repair. Curr Opin Organ Transplant. 2010;15:86-91.

[8] Verseijden F, Posthumus-van Sluijs SJ, Pavljasevic P, Hofer SO, van Osch GJ, Farrell E. Adult human bone marrow- and adipose tissue-derived stromal cells support the formation of prevascular-like structures from endothelial cells in vitro. Tissue Eng Part A. 2010;16: 101-14.

[9] Pascual I, de Miguel GF, Gomez-Pinedo UA, de Miguel F, Arranz MG, Garcia-Olmo D. Adipose-derived mesenchymal stem cells in biosutures do not improve healing of experimental colonic anastomoses. Br J Surg. 2008;95:1180-4.

[10] Yoo JH, Shin JH, An MS, Ha TK, Kim KH, Bae KB, et al. Adipose-tissue-derived Stem Cells Enhance the Healing of Ischemic Colonic Anastomoses: An Experimental Study in Rats. Journal of the Korean Society of Coloproctology. 2012;28:132-9.

[11] Yang J, Yamato M, Shimizu T, Sekine H, Ohashi K, Kanzaki M, et al. Reconstruction of functional tissues with cell sheet engineering. Biomaterials. 2007;28:5033-43.

[12] Matsuda N, Shimizu T, Yamato M, Okano T. Tissue Engineering Based on Cell Sheet Technology. Advanced Materials. 2007;19:3089-99.

[13] Daams F, Luyer M, Lange JF. Colorectal anastomotic leakage: aspects of prevention, detection and treatment. World J Gastroenterol. 2013;19:2293-7.

[14] Hamdi H, Planat-Benard V, Bel A, Puymirat E, Geha R, Pidial L, et al. Epicardial adipose stem cell sheets results in greater post-infarction survival than intramyocardial injections. Cardiovasc Res. 2011;91:483-91.

[15] Hamdi H, Boitard SE, Planat-Benard V, Pouly J, Neamatalla H, Joanne P, et al. Efficacy of epicardially delivered adipose stroma cell sheets in dilated cardiomyopathy. Cardiovasc Res. 2013;99:640-7.

[16] Makarevich PI, Boldyreva MA, Gluhanyuk EV, Efimenko AY, Dergilev KV, Shevchenko EK, et al. Enhanced angiogenesis in ischemic skeletal muscle after transplantation of cell sheets from baculovirus-transduced adipose-derived stromal cells expressing VEGF165. Stem Cell Res Ther. 2015;6:204.

[17] Kato Y, Iwata T, Morikawa S, Yamato M, Okano T, Uchigata Y. Allogeneic transplantation of an adipose-derived stem cell (ASC) sheet combined with artificial skin accelerates wound healing in a rat wound model of type 2 diabetes and obesity. Diabetes. 2015.

[18] van Buul GM, Farrell E, Kops N, van Tiel ST, Bos PK, Weinans H, et al. Ferumoxides-protamine sulfate is more effective than ferucarbotran for cell labeling: implications for clinically applicable cell tracking using MRI. Contrast Media Mol Imaging. 2009;4:230-6.

[19] Wu Z, Daams F, Boersema GS, Vakalopoulos KA, Lam KH, van der Horst PH, et al. Colorectal anastomotic leakage caused by insufficient suturing after partial colectomy: a new experimental model. Surg Infect (Larchmt). 2014;15:733-8.

[20] Lin CS, Lin G, Lue TF. Allogeneic and xenogeneic transplantation of adipose-derived stem cells in immunocompetent recipients without immunosuppressants. Stem Cells Dev. 2012;21:2770-8. 
[21] Verco SJ, Peers EM, Brown CB, Rodgers KE, Roda N, diZerega G. Development of a novel glucose polymer solution (icodextrin) for adhesion prevention: pre-clinical studies. Hum Reprod. 2000;15:1764-72.

[22] Yang J, Yamato $M$, Shimizu T, Sekine $H$, Ohashi K, Kanzaki M, et al. Reconstruction of functional tissues with cell sheet engineering. Biomaterials. 2007;28:5033-43.

[23] Shimizu T, Yamato M, Kikuchi A, Okano T. Cell sheet engineering for myocardial tissue reconstruction. Biomaterials. 2003;24:2309-16.

[24] Perrod G, Rahmi G, Pidial L, Camilleri S, Bellucci A, Casanova A, et al. Cell Sheet Transplantation for Esophageal Stricture Prevention after Endoscopic Submucosal Dissection in a Porcine Model. PLoS One. 2016;11:e0148249.

[25] Hamdi H, Planat-Benard V, Bel A, Puymirat E, Geha R, Pidial L, et al. Epicardial adipose stem cell sheets results in greater post-infarction survival than intramyocardial injections. Cardiovasc Res. 2011;91:483-91.

[26] Aysan E, Dincel O, Bektas H, Alkan M. Polypropylene mesh covered colonic anastomosis. Results of a new anastomosis technique. Int J Surg. 2008;6:224-9.

[27] Wu Z, Vakalopoulos KA, Kroese LF, Boersema GS, Kleinrensink GJ, Jeekel J, et al. Reducing anastomotic leakage by reinforcement of colorectal anastomosis with cyanoacrylate glue. Eur Surg Res. 2013;50:255-61.

[28] Sukho P, Kirpensteijn J, Hesselink JW, van Osch GJ, Verseijden F, Bastiaansen-Jenniskens YM. Effect of Cell Seeding Density and Inflammatory Cytokines on Adipose TissueDerived Stem Cells: an in Vitro Study. Stem Cell Rev. 2017.

[29] Shudo Y, Miyagawa S, Ohkura H, Fukushima S, Saito A, Shiozaki M, et al. Addition of mesenchymal stem cells enhances the therapeutic effects of skeletal myoblast cell-sheet transplantation in a rat ischemic cardiomyopathy model. Tissue Eng Part A. 2014;20: 728-39.

[30] Otsuki Y, Nakamura Y, Harada S, Yamamoto Y, Ogino K, Morikawa K, et al. Adipose stem cell sheets improved cardiac function in the rat myocardial infarction, but did not alter cardiac contractile responses to beta-adrenergic stimulation. Biomed Res. 2015;36:11-9.

[31] Pommergaard HC, Achiam MP, Rosenberg J. External coating of colonic anastomoses: a systematic review. International journal of colorectal disease. 2012;27:1247-58.

[32] Suarez-Grau JM, Bernardos Garcia C, Cepeda Franco C, Mendez Garcia C, Garcia Ruiz S, Docobo Durantez F, et al. Fibrinogen-thrombin collagen patch reinforcement of highrisk colonic anastomoses in rats. World J Gastrointest Surg. 2016;8:627-33.

[33] Liu L, Chiu PW, Lam PK, Poon CC, Lam CC, Ng EK, et al. Effect of local injection of mesenchymal stem cells on healing of sutured gastric perforation in an experimental model. Br J Surg. 2015;102:e158-68.

[34] Yu J, Tu YK, Tang YB, Cheng NC. Stemness and transdifferentiation of adipose-derived stem cells using L-ascorbic acid 2-phosphate-induced cell sheet formation. Biomaterials. 2014;35:3516-26.

[35] Alexeev V, Arita M, Donahue A, Bonaldo P, Chu M-L, Igoucheva O. Human adipose-derived stem cell transplantation as a potential therapy for collagen VI-related congenital muscular dystrophy. Stem Cell Res Ther. 2014;5:21-.

[36] Rabau MY, Hirshberg A, Hiss Y, Dayan D. Intestinal anastomosis healing in rat: collagen concentration and histochemical characterization by Picrosirius red staining and polarizing microscopy. Exp Mol Pathol. 1995;62:160-5.

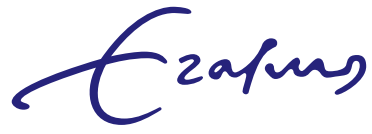


[37] Leoni G, Neumann PA, Sumagin R, Denning TL, Nusrat A. Wound repair: role of immuneepithelial interactions. Mucosal Immunol. 2015;8:959-68.

[38] Anderson P, Souza-Moreira L, Morell M, Caro M, O’Valle F, Gonzalez-Rey E, et al. Adiposederived mesenchymal stromal cells induce immunomodulatory macrophages which protect from experimental colitis and sepsis. Gut. 2013;62:1131-41.

[39] Ishikane S, Hosoda H, Yamahara K, Akitake Y, Kyoungsook J, Mishima K, et al. Allogeneic transplantation of fetal membrane-derived mesenchymal stem cell sheets increases neovascularization and improves cardiac function after myocardial infarction in rats. Transplantation. 2013;96:697-706.

[40] Schaffer M, Barbul A. Lymphocyte function in wound healing and following injury. Br J Surg. 1998;85:444-60.

[41] Meyerrose TE, De Ugarte DA, Hofling AA, Herrbrich PE, Cordonnier TD, Shultz LD, et al. In vivo distribution of human adipose-derived mesenchymal stem cells in novel xenotransplantation models. Stem Cells. 2007;25:220-7.

[42] Ashkanani F, Krukowski ZH. Intestinal Anastomosis. Surgery (Oxford). 2002;20:104-7.

\section{SUPPORTING METHODS}

\section{ASCs isolation and culture}

Human ASCs were isolated from subcutaneous abdominal adipose tissue from eight healthy female donors (age 25-50 years) as leftover adipose tissue from breast reconstruction surgery, with approval of the Medical Ethical Committee of the Erasmus Medical Center, Rotterdam (MEC-2014-092). ASCs were isolated as described previously.[8] Briefly, after digestion of the tissue, ASCs were plated in 40,000 cells/ $\mathrm{cm}^{2}$ and grown in Dulbecco's Modified Eagle Medium $1 \mathrm{~g} / \mathrm{l}$ glucose (LGDMEM, Gibco, Life technologies, Paisley, UK), containing $20 \%$ fetal bovine serum (FBS, Lonza, Verviers, Belgium), 50 g g/ml gentamicin (Gibco), $1.5 \mu \mathrm{g} / \mathrm{mL}$ fungizone ${ }^{\circledR}$ (Gibco). Ascorbic acid-2-phosphate ( $25 \mu \mathrm{g} / \mathrm{ml}$, Sigma-Aldrich, St. Louis, MO, USA) and $1 \mathrm{ng} / \mathrm{ml}$ human recombinant fibroblast growth factor 2 (FGF2, AbDSerotec, Kidlington, UK)] were freshly added. After 24 hours, cultures were washed with Dulbecco's phosphate-buffered saline (PBS, Gibco) with 2\% FBS to remove nonadherent cells and erythrocytes and refreshed with expansion medium (LG-DMEM with $10 \%$ FBS, $50 \mu \mathrm{g} / \mathrm{ml}$ gentamicin, $1.5 \mu \mathrm{g} / \mathrm{mL}$ fungizone ${ }^{\circledR}$ and freshly added ascorbic acid-2-phosphate and FGF2). Isolated ASCs were cultured at $37^{\circ} \mathrm{C}$ under humidified conditions in an atmosphere with $5 \% \mathrm{CO}_{2}$. When cultures reached $90 \%$ confluence, ASCs were sub-cultured with $0.25 \%$ trypsin EDTA (Gibco) and reseeded at a density of 8,000 cells $/ \mathrm{cm}^{2}$ for two passages and thereafter. ASCs at passages 2-5 were used for following experiments.

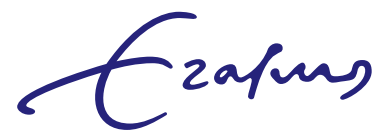




\section{Immunohistochemistry}

All sections were treated with xylene and ethanol series to deparaffinize. Heat induced antigen retrieval was used by boiling sections at $95{ }^{\circ} \mathrm{C}$ for 20 minutes in citrate buffer or Tris-EDTA buffer solution. Vascular density was evaluated using antibodies against CD34 (AF4117, dilution 1:200; R\&D systems, Minneapolis, Minnesota). T-cells were identified by CD3 staining (Ab16669, dilution 1:100; ab16669; Abcam Cambridge, UK). M2 macrophages were identified by CD163 staining (MCA3426A, dilution 1:100; AbDSerotec, Raleigh, NC, USA). Anti-human mitochondria antibody (Abcam AB92824, dilution 1:500, Cambridge, UK) was used to identify viable ASC sheet. Human endothelial cells were identified with a mouse anti human antibody against CD31 (M0823, dilution 1:100, Dako, Glostrup, Denmark). After blocking with normal goat serum each primary antibody was diluted in $1 \%$ bovine serum albumin in PBS and sections were incubated for 60 minutes at room temperature. The slides were then incubated with biotinylated goat-antirabbit link (BioGenex Laboratories, San Ramon, CA, USA) for 30 minutes followed by incubation with streptavidin-AP (Biogenex) for 30 minutes. Neu Fuchsin (Chroma, Kongen, Germany), napthtol AS-MX phosphate (Sigma), sodium nitrate (Sigma), levamisole (Sigma) and dimethyformamid (Sigma) were used in the substrate. Hematoxylin was used as a counter stain. Isotype-matched antibodies were used as negative controls.

The histological and immunohistochemical assessments were performed by two independent blinded observers (PS and AC). Seven images of each slide were taken for analysis including the anastomotic area and adjacent area in every layer. The number of positive cells for each photograph from high power field (200x magnification, Olympus BX50, Olympus, Hamburg, Germany) was counted with ImageJ (National Institutes of Health, Bethesda, MD). For CD3 and CD163, the values were expressed as average number of positive cells per $\mathrm{mm}^{2}$. For capillary density, values were expressed as average number of $\mathrm{CD} 34$ positive vessels per $\mathrm{mm}^{2}$. Quantification of collagen deposition within the anastomotic healing area was calculated as percentage of Picrosirius red positive staining area. Two sections were randomly selected from each slide and 3 images of the anastomotic area were evaluated using Image J software.

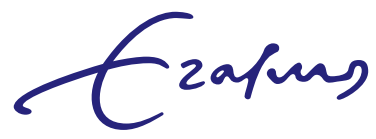




\section{SUPPLEMENTARY RESULTS}

A Postoperative weight change

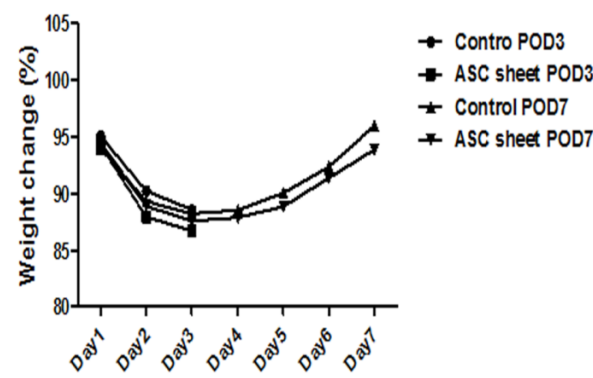

B Postoperative wellness score

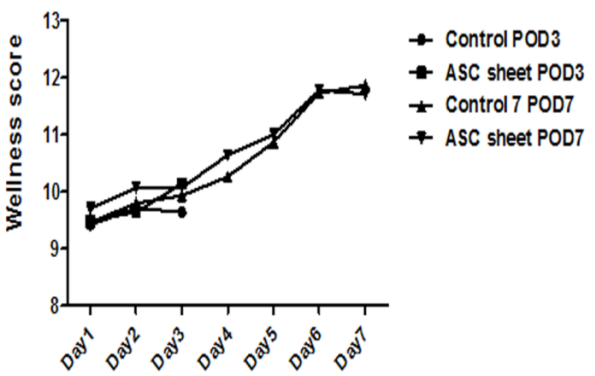

Supplementary Figure 1. (A) Postoperative weight changes and (B) wellness score (0-12). $\mathrm{ASC}=$ adipose tissue-derived stem cells. $\mathrm{POD} 3=3$ days post operation, $\mathrm{POD} 7=7$ days post operation.

A Abscess number elsewhere

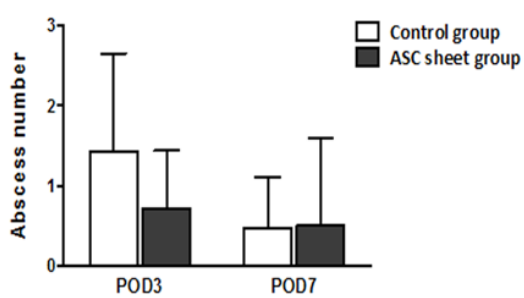

$\mathrm{C}_{\text {Adhesion number elsewhere }}$

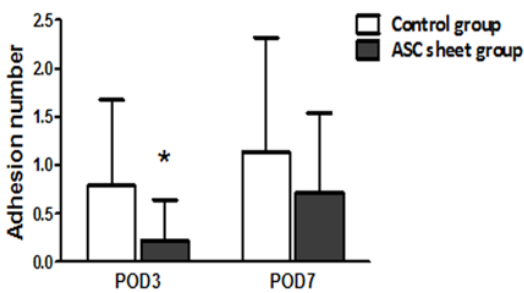

B Abscess score elsewhere

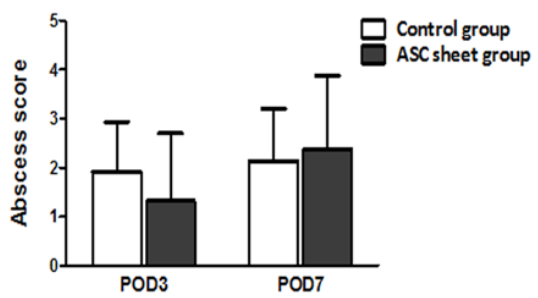

D Adhesion score elsewhere

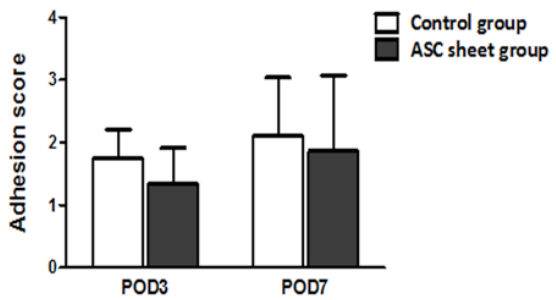

Supplementary Figure 2. Intra-abdominal macroscopic findings distant from colorectal anastomosis. (A) Abscess number, (B) abscess score (0-4), (C) adhesion number and (D) adhesion score (0-4).

ASC $=$ adipose tissue-derived stem cells. POD3 $=$ postoperative day 3. POD7 $=$ postoperative day 7. Each bar represents average number $+/$ - standard deviation from control $(n=14$ in the control and ASC sheet group at POD3; $n=15$ in the control group and $n=14$ in the ASC sheet group at POD7), ${ }^{*} P<0.05$ between groups. 